\title{
Jointly Optimal Downlink Beamforming and Base Station Assignment
}

\author{
In proceedings of ICASSP - 2001 \\ DOI: 10.1109/ICASSP.2001.940270
}

(C) 2001 IEEE. Personal use of this material is permitted. However, permission to reprint/republish this material for advertising or promotional purposes or for creating new collective works for resale or redistribution to servers or lists, or to reuse any copyrighted component of this work in other works must be obtained from the IEEE.

\section{MATS BENGTSSON}

IR-S3-SB-0112 


\title{
JOINTLY OPTIMAL DOWNLINK BEAMFORMING AND BASE STATION ASSIGNMENT
}

\author{
Mats Bengtsson \\ Signal Processing, S3, \\ Royal Institute of Technology (KTH) \\ 10044 Stockholm, SWEDEN \\ Tel: +4687908463, fax: +4687907260 \\ Email: matsbes3.kth.se
}

\begin{abstract}
We present an algorithm that jointly determines the optimal downlink beamformers and the optimal assignment of each mobile to a base station. The optimality criterion is based on a systems perspective; provide sufficient quality of service for all users, transmitting as little excess power as possible. Since the algorithm is centralized and requires knowledge about all the channels in the system, it may be infeasible in a practical implementation. However, it provides the ultimate benchmark in system evaluations. Numerical examples show substantial gain compared to ordinary base station assignment.
\end{abstract}

\section{INTRODUCTION}

Uplink beamforming is a well-established field with a large literature available describing algorithms and performance analysis. Downlink beamforming in a communications system, on the other hand, has only received any major attention during the last years. Different methods were suggested in, for example, $[1,2,3,4]$ but the problem of optimal downlink beamforming, as formulated in [5] was first solved by RashidFarrokhi et.al. [6] who presented a generalization of the standard power control algorithm which always will converge to the optimal solution if the problem is feasible. A similar algorithm was derived independently in [7] and an alternative algorithm based on semidefinite optimization was presented in $[8,9]$.

For the problem of assigning each mobile to a base station, an algorithm is presented in [10] for uplink communication which jointly determines the optimal uplink beamformers at the base station, the power control of each mobile and the base station assignment. Again, the algorithm is based on a corresponding algorithm for single-antenna systems [11]. When it comes to jointly optimal downlink beamforming and base station assignment, no general solution has been presented, to our knowledge, even for the case of single-antenna base stations. In [12], it is shown that the problem is not Pareto optimal, i.e., the assignment that is best for the system is not necessarily the best for each individual link.
Here, we will present algorithms to determine the jointly optimal downlink beamformers and assignment of mobiles to base stations, for a set of co-channel users. The algorithm is based on global knowledge about the channels from all base stations to all mobiles. In a Frequency Duplex Division (FDD) system, the uplink and the downlink channels will typically fade independently which means that the instantaneous downlink channel can never be estimated from uplink measurements. However, it may still be reasonable to estimate the second-order statistics of the fast fading of the downlink channel based on uplink measurements averaged over the fast fading. Several methods have been proposed to compensate for the frequency offset between the uplink and downlink channels, see for example $[4,13,14]$ and will not be described further here. The resulting solution will optimize the average performance over the fast fading.

We first review the solutions for optimal downlink beamforming with a given base station assignment and then show how to extend the methods to provide also the optimal assignment. Finally, we present some numerical examples and conclusions.

\section{MODEL AND NOTATION}

We consider a system where a number of co-channel mobile users are served by one or more base stations and each base station is equipped with an antenna array. We will design the beamformers assuming a stationary scenario where the fast (Rayleigh) fading is described by its second order properties. We also assume narrow-band signals without any time dispersion, i.e., the channel fading is frequency flat. The model can easily be extended to frequency selective channels, taking both co-channel interference and inter-symbol interference into account, see [6, 14].

In the baseband, the signal received by the $i$ th mobile, $r_{i}(t)$, is given by

$$
r_{i}(t)=\sum_{k=1}^{K} \mathbf{h}_{i, k}^{*} \mathbf{x}_{k}(t)+n_{i}(t),
$$


where $(\cdot)^{*}$ denotes Hermitian vector transpose. Here, $\mathbf{x}_{k}(t)$ is the complex valued $m \times 1$ vector of the baseband signals transmitted at the antenna elements of base station $k, n_{i}(t)$ is zero mean white complex noise with variance $\sigma_{i}^{2}$. The channel from base station $k$ to mobile $i$ is given by the random complex valued vector $\mathbf{h}_{i, k}$ with correlation matrix

$$
\mathbf{R}_{i, k}=\mathrm{E}\left[\mathbf{h}_{i, k} \mathbf{h}_{i, k}^{*}\right] .
$$

In the special case of line of sight transmission, $\mathbf{R}_{i, k}=\mathbf{h}_{i, k} \mathbf{h}_{i, k}^{*}$, where $\mathbf{h}_{i, k}$ is a deterministic array response vector, but in general, $\mathbf{R}_{i, k}$ can have any rank because of specular or diffuse multi-path propagation.

Each mobile is assigned to one base station and $\kappa_{i}$ is used to denote the base station assigned for mobile $i$. Conversely, $\mathcal{I}(k)=\left\{i \mid \kappa_{i}=k\right\}$ denotes the indices of the set of mobiles assigned to base $k$.

The signal transmitted at base $k$ is given by

$$
\mathbf{x}_{k}(t)=\sum_{i \in \mathcal{I}(k)} \mathbf{w}_{i} s_{i}(t)
$$

where $s_{i}(t)$ is the scalar modulated data sequence intended for user $i$ and $\mathbf{w}_{i}$ is the beamforming weight vector for transmission from base $\kappa_{i}$ to mobile $i$. For simplicity we assume that all $s_{i}(t)$ are uncorrelated and have the same normalized power $\mathrm{E}\left[\left|s_{i}(t)\right|^{2}\right]=1$.

\section{OPTIMAL DOWNLINK BEAMFORMING}

We first consider the design of the beamformers $\mathbf{w}_{i}$ given estimates of all $\mathbf{R}_{i, k}$ and $\sigma_{i}^{2}$ for a given assignment $\kappa_{i}$. The goal is to minimize to total transmitted power

$$
P=\sum_{i=1}^{d}\left\|\mathbf{w}_{i}\right\|^{2}
$$

while maintaining an acceptable quality of service (QoS) for all users. The QoS requirement is specified in terms of a lower threshold, $\operatorname{SINR}_{i} \geq \gamma_{i}$, of the received SINR at each mobile. This gives the following optimization problem

$$
\begin{aligned}
& \min \sum_{i=1}^{d}\left\|\mathbf{w}_{i}\right\|^{2} \\
& \text { s.t. } \frac{\mathbf{w}_{i}^{*} \mathbf{R}_{i} \mathbf{w}_{i}}{\sum_{n \neq i} \mathbf{w}_{n}^{*} \mathbf{R}_{i, \kappa_{n}} \mathbf{w}_{n}+\sigma_{i}^{2}} \geq \gamma_{i}, i=1, \ldots, d,
\end{aligned}
$$

or equivalently,

$$
\begin{aligned}
& \min \sum_{i=1}^{d} \mathbf{w}_{i}^{*} \mathbf{w}_{i} \\
& \text { s.t. } \mathbf{w}_{i}^{*} \mathbf{R}_{i} \mathbf{w}_{i}-\gamma_{i} \sum_{n \neq i} \mathbf{w}_{n}^{*} \mathbf{R}_{i, \kappa_{n}} \mathbf{w}_{n} \geq \gamma_{i} \sigma_{i}^{2}, i=1, \ldots, d .
\end{aligned}
$$

It is easy to show that all constraints must be active at the optimum [7], thus the inequality in (6) can be replaced by an equality.

In [6], it is (implicitly) shown that the solutions of (6) and of the following problem,

$$
\begin{aligned}
\min _{\mathbf{u}_{i}, \rho_{i}} & \sum_{n=1}^{d} \rho_{n} \\
\text { s.t. } & \frac{\mathbf{u}_{i}^{*} \rho_{i} \mathbf{R}_{i, \kappa_{i}} \mathbf{u}_{i}}{\mathbf{u}_{i}^{*}\left(\sum_{n \neq i} \rho_{n} \gamma_{n} \mathbf{R}_{n, \kappa_{i}}+\mathbf{I}\right) \mathbf{u}_{i}} \geq 1 \\
& \left\|\mathbf{u}_{i}\right\|^{2}=1,
\end{aligned}
$$

are related by $\mathbf{w}_{i}=\sqrt{p_{i}} \mathbf{u}_{i}$ for some power levels $p_{i}$. The values of $p_{i}$ can be determined uniquely from a linear system of equations that gives equality in all the constraints of (6). Note that (7) has exactly the same form as an uplink beamforming problem to determine the optimal receiving beamformers $\mathbf{u}_{i}$ at the base stations and power levels $\rho_{i}$ at the mobiles. However, since the true uplink problem for the system differs from (7), it is called the virtual uplink problem. In [10], it is shown that such an uplink problem has a unique solution which can be determined using an extended power control loop, which in each iteration finds the optimal beamformers given the power levels and then makes a standard power control update to improve the power levels. Just as the standard power control loop, the algorithm can be implemented in a distributed fashion, where each base station updates its own beamformers and informs the corresponding mobiles based only on information available locally at the base station. This algorithm can be applied to the virtual uplink problem (although not in a distributed implementation) and will, followed by downlink power control, provide the optimal solution of (5), see [6].

\section{BEAMFORMING AND BASE STATION ASSIGNMENT}

The algorithm for jointly optimal downlink beamforming and assignment of mobiles to base stations can be derived in several ways. The goal is to optimize (5) not only over the beamforming vectors $\mathbf{w}_{i}$ but also over the assignment $\kappa_{i}$. A conceptually interesting route is to relax the problem and allow for all base stations to transmit to all the mobiles at the same time. In other words, we view all the base station arrays as part of a single giant base station antenna array that transmits to all the co-channel users. However, we do not allow for coherent transmission from different base stations. This can be included into the model by setting $\mathrm{E}\left[\mathbf{h}_{i, k_{1}} \mathbf{h}_{i, k_{2}}^{*}\right]=0$ for all $k_{1} \neq k_{2}$. Note that the desired scenario, where each mobile is served by a single base station is included in this extended model as a special case. The optimal downlink beamformers $\overline{\mathbf{w}}_{i}=\left[\mathbf{w}_{i, 1}^{T}, \ldots, \mathbf{w}_{i, K}^{T}\right]^{T}$ from this single giant base station can be found by solving (5) for the single base station case 
with the channel correlation matrix, from the giant combined base station to all the mobiles, given by

$$
\overline{\mathbf{R}}_{i}=\left[\begin{array}{cccc}
\mathbf{R}_{i, 1} & 0 & \ldots & 0 \\
0 & \mathbf{R}_{i, 2} & \ddots & \vdots \\
\vdots & \ddots & \ddots & 0 \\
0 & \ldots & 0 & \mathbf{R}_{i, d}
\end{array}\right] .
$$

Analyzing the algorithm of [6], it turns out that there is no need to implement and store all the zeros of (8). Also, it turns out that for each signal $i$, only one of the $\mathbf{w}_{i, k}$ is nonzero. The interesting conclusion is that even with this relaxed scenario which allows every base station to communicate with each mobile, the optimal solution is actually to assign each mobile to a single base station $\left(\kappa_{i}=\right.$ the $k$ index of the nonzero $\mathbf{w}_{i, k}$ ) and use the beamformer $\mathbf{w}_{i}=\mathbf{w}_{i, \kappa_{i}}$. The resulting algorithm can be summarized in the following steps (index $t$ is here used as an iteration counter, not to be confused with any time index of the channel samples).

1. Initialize $\rho_{i}(1)=1$ for $i=1,2, \ldots, d$ (or use some other non-negative initial value).

2. For $t=1,2, \ldots$ until convergence, iterate the following steps

Update of candidate optimal beamformers: For each $i=1,2, \ldots, d$ and $k=1,2, \ldots, K$, find

$$
\mu_{i, k}=\max _{\|\mathbf{u}\|=1} \frac{\rho_{i}(t) \mathbf{u}^{*} \mathbf{R}_{i, k} \mathbf{u}}{\mathbf{u}^{*}\left(\sum_{n \neq i} \rho_{n}(t) \gamma_{n} \mathbf{R}_{n, k}+\mathbf{I}\right) \mathbf{u}}
$$

and call the corresponding vector $\mathbf{u}_{i, k}$.

Assignment update: For each $i=1,2, \ldots, d$,

$$
\kappa_{i}=\underset{1 \leq k \leq K}{\arg \max } \mu_{i, k}
$$

Power control update: Let

$$
\rho_{i}(t+1)=\frac{\gamma_{i}}{\mu_{i, \kappa_{i}}} \rho_{i}(t)
$$

3. After convergence,

$$
\begin{aligned}
\boldsymbol{\eta} & =\left[\gamma_{1} \sigma_{1}^{2}, \ldots, \gamma_{d} \sigma_{d}^{2}\right]^{T} \\
{[\mathbf{F}]_{i, n} } & = \begin{cases}\mathbf{u}_{i, \kappa_{i}}^{*} \mathbf{R}_{i} \mathbf{u}_{i, \kappa_{i}} & i=n \\
-\gamma_{i} \mathbf{u}_{n, \kappa_{n}}^{*} \mathbf{R}_{i \kappa_{n}} \mathbf{u}_{n, \kappa_{n}} & i \neq n\end{cases} \\
\mathbf{p} & =\mathbf{F}^{-1} \boldsymbol{\eta} \\
\mathbf{w}_{i} & =\sqrt{[\mathbf{p}]_{i}} \mathbf{u}_{i, \kappa_{i}}
\end{aligned}
$$

Note that the maximum $\mu_{i, k}$ and $\mathbf{u}_{i, k}$ in (9) are given by the maximum eigenvalue and the corresponding eigenvalue, respectively, of the generalized eigenvalue problem

$$
\rho_{i}(t) \mathbf{R}_{i, k} \mathbf{u}_{i, k}=\mu_{i}\left(\sum_{n \neq i} \rho_{n}(t) \gamma_{n} \mathbf{R}_{n, k}+\mathbf{I}\right) \mathbf{u}_{i, k} .
$$

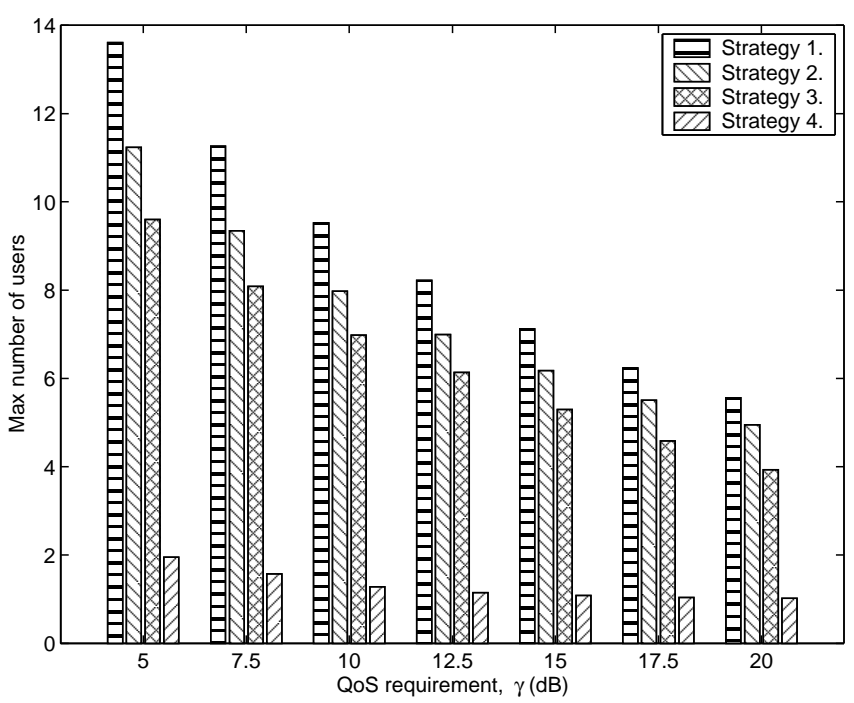

Fig. 1. Maximum number of co-channel users in the system for different QoS requirements and downlink strategies.

The first two steps of the algorithm are identical to the uplink algorithm for joint beamforming, power control and base assignment from [10], applied to the virtual uplink problem (7). Of course, this observation could be used directly to derive our algorithm. An alternative algorithm, with larger flexibility to handle additional constraints, can be derived based on the semidefinite programming downlink algorithm of [9, 8], using the same relaxation principle.

\section{NUMERICAL EXAMPLES}

With a small simulated scenario, we illustrate the type of results that could be obtained in terms of system performance. The setup is a narrowband system with 4 base stations, each equipped with an 8 element uniform circular array with a radius of one wavelength. We try to add as many co-channel users as possible, maintaining a specific QoS level (SINR $\geq$ $\gamma$ ) for all users. Each mobile is placed at random in the area and the propagation is affected by log-normal shadow fading and diffuse multipath scattering. The following four strategies were compared:

1. Jointly optimal beamforming and base station assignment.

2. Optimal beamforming but a fixed assignment, where each terminal is assigned to the base station with the highest path gain.

3. A decentralized interference suppressing beamformer similar to the one in $[3,15,4]$ combined with standard power control, using the same fixed assignment. 
4. Conventional beamforming without interference suppression, using the same assignment and standard power control.

Figure 1 shows the maximum number of users that could be admitted to the system, providing all users with the required SINR level, for the different strategies and for different SINR requirements. The results in the figure are averaged over 300 random scenarios.

The additional gain of the optimal assignment was 12 $20 \%$ in this experiment and may be even higher in other situations. The decentralized beamforming algorithm provides almost the same performance as the optimal beamformer but the results are sensitive to the choice of a regularization parameter, see [14]. During the experiments, we made the interesting observation that the optimal assignment may change with the SINR requirement, $\gamma_{i}$.

\section{CONCLUSIONS}

We have presented a new algorithm that determines the optimal assignment of mobiles to base station and downlink beamformers at the base stations, for a set of co-channel users. Even though the optimization problem involves both combinatorial and non-convex quadratic constraints, it can be solved efficiently. Several different algorithms can be used, but because of the limited space, we presented one specific algorithm based on the previous work by Rashid-Farrokhi at.al. [6, 10] and outlined how to extend any downlink beamforming algorithm to provide also the optimal assignment.

The solution requires global knowledge about all channels involved in the system (at least the second order statistics of the fast fading for each channel) and may for that reason be primarily of interest as a benchmark in system simulations. The gain compared to other assignment schemes may be significant and an interesting topic for future research is to find decentralized base station assignment schemes that can approach the optimal solution.

\section{References}

[1] Derek Gerlach and Arogyaswami Paulraj, "Adaptive transmitting antenna arrays with feedback," IEEE SP Letters, vol. 1, no. 10, pp. 150-152, Oct. 1994.

[2] Gregory G. Raleigh, Suhas N. Diggavi, V. K. Jones, and A. Paulraj, "A blind adaptive transmit antenna algorithm for wireless communication," in Proc. ICC. IEEE, 1995, vol. 3, pp. 1494-1499.

[3] Per Zetterberg and Björn Ottersten, "The spectrum efficiency of a base station antenna array for spatially selective transmission," IEEE Trans. VT, vol. 44, pp. 651-660, Aug. 1995.

[4] Thierry Asté, Philippe Forster, Luc Féty, and Sylvie Mayrargue, "Downlink beamforming for cellular mobile communications (GSM system)," Annales des Télécommunications, vol. 53, no. 11-12, pp. 435-448, Nov.-Dec. 1998.
[5] Christof Farsakh and Josef A. Nossek, "Channel allocation and downlink beamforming in an SDMA mobile radio system," in Proc. PIMRC. IEEE, 1995, vol. 2, pp. 687-691.

[6] Farrokh Rashid-Farrokhi, K.J. Ray Liu, and Leandros Tassiulas, "Transmit beamforming and power control for cellular wireless systems," IEEE J. Select. Areas Commun., vol. 16, no. 8, pp. 1437-1450, Oct. 1998.

[7] Eugene Visotsky and Upamanyu Madhow, "Optimum beamforming using transmit antenna arrays," in Proc. VTC'99, Houston, Texas, USA, May 1999, IEEE, vol. 1, pp. 851-856.

[8] Mats Bengtsson and Björn Ottersten, "Optimal downlink beamforming using semidefinite optimization," in Proc. 37th Annual Allerton Conference on Communication, Control, and Computing, Sept. 1999, pp. 987-996.

[9] Mats Bengtsson, Antenna Array Signal Processing for High Rank Data Models, Ph.D. thesis, Royal Institute of Technology, Stockholm, Sweden, Dec. 1999, TRITA-S3-SB-9938.

[10] Farrokh Rashid-Farrokhi, Leandros Tassiulas, and K.J. Ray Liu, "Joint optimal power control and beamforming in wireless networks using antenna arrays," IEEE Trans. Commun., vol. 46, no. 10, pp. 1313-1324, Oct. 1998.

[11] Roy D. Yates, "A framework for uplink power control in cellular radio systems," IEEE J. Select. Areas Commun., vol. 13, no. 7, pp. 1341-1347, Sept. 1995.

[12] Farrokh Rashid-Farrokhi, K.J. Ray Liu, and Leandros Tassiulas, "Downlink power control and base station assignment," IEEE Communications Letters, vol. 1, no. 4, pp. 102-104, July 1997.

[13] Klaus Hugl, Juha Laurila, and Ernst Bonek, "Downlink beamforming for frequency division duplex systems," in Proc. GLOBECOM, 1999, vol. 4, pp. 2097-2101.

[14] Mats Bengtsson and Björn Ottersten, "Optimal and suboptimal transmit beamforming," in Handbook of Antennas in Wireless Communications, Lal C. Godara, Ed., chapter 18. CRC Press, Aug. 2001.

[15] Per Zetterberg, "A comparison of two systems for downlink communication with base station antenna arrays," IEEE Trans. $V T$, vol. 48, no. 5, pp. 1356-1370, Sept. 1999. 